\title{
Feeding-Mediated Distention Inhibits Swimming in the Medicinal Leech
}

\author{
Quentin Gaudry ${ }^{1}$ and William B. Kristan Jr ${ }^{2}$ \\ ${ }^{1}$ Neurosciences Graduate Program and ${ }^{2}$ Neurobiology Section, Division of Biological Sciences, University of California, San Diego, La Jolla, California \\ 92093-0357
}

An animal's response to a stimulus depends on many factors such as age, hormonal state, experience, and its behavioral state. For example, an animal may suppress a behavior that is inappropriate or incompatible with its current state. In this study, we show that, as a medicinal leech feeds, the distention that it incurs inhibits its expression of swimming. Distention slows the swimming pattern and decreases the number of swim cycles elicited by a test electrical stimulation; large distentions inhibit swimming altogether. We have previously shown that the ingestive phase of feeding inhibits behaviors by presynaptic inhibition of mechanosensory neurons. Distention has its effects downstream (e.g., gating and central pattern generating interneurons) from these sensory neurons and thus represents a novel mechanism for choosing between conflicting behaviors during feeding. Because removing the leech's gut surgically did not eliminate the effects of body distention, we conclude that the receptors mediating the distention-induced suppression of swimming are likely to be located in the animal's body wall. Together with previous findings, these new data show that leeches rely on two different decisionmaking networks to ensure that a biologically important behavior is not disrupted by other behaviors.

\section{Introduction}

In the CNS, decision making involves the competition among neuronal networks to select the most appropriate behavioral output for the task at hand. The interactions between any two behavioral circuits can occur in early sensory processing, between interneurons that command the two behaviors, or between the pattern-generating circuits that drive the motor patterns. Understanding how such competing circuits interact within the CNS is essential to understanding how decisions are generated. Here, we show that the decision to abstain from swimming during feeding occurs at multiple levels within the leech CNS.

In complex brains, such as those of mammals, different brain regions (e.g., visual, auditory, and somatosensory cortices) are specialized for particular tasks (Kandel et al., 2000). In mammals, higher-level cognitive functions like decision making rely on interactions among multiple brain regions. For instance, distinct areas of the superior colliculus encode when and where to make visual saccades (Glimcher and Sparks, 1992; Glimcher, 2001); primates store reward probability knowledge in the lateral intraparietal area (LIP), whereas the basal ganglia encodes information about the reward value (Schultz, 1998; Platt and Glimcher, 1999; Glimcher, 2003); and human functional magnetic resonance imaging studies have shown that different brain structures are involved in different steps used in

\footnotetext{
Received March 23, 2010; revised May 25, 2010; accepted June 2, 2010.

This work was supported by National Institutes of Health Grants MH43396 and NS35336 (W.B.K.) and by National Science Foundation Grant IOB-523959 (W.B.K., Karen A. Mesce). We thank Natalie Ruiz for assistance with experiments. Q.G. thanks Kate Gaudry for assistance with data analysis. We also thank Krista Todd for her expertise in inverting leeches.

Correspondence should be addressed to William B. Kristan Jr, Neurobiology Section, Division of Biological Sciences, University of California, San Diego, 3119 Pacific Hall, 9500 Gilman Drive, \#0357, La Jolla, CA 92093-0357. E-mail:wkristan@ucsd.edu.

DOI:10.1523/JNEUROSCI.1487-10.2010

Copyright $\odot 2010$ the authors $\quad 0270-6474 / 10 / 309753-09 \$ 15.00 / 0$
}

economic decision making (Liu et al., 2007; Hare et al., 2008). These distributed circuits are also likely to be modularized, with the same structures being recruited to perform a specific function that is commonly involved in multiple tasks. Receiving either monetary or social gains, for example, recruits overlapping reward regions in human brains (Izuma et al., 2008).

To determine how distributed and modularized decisionmaking circuits are organized at the cellular level, one needs to monitor both behavior and brain activity simultaneously. The simple nervous systems of invertebrates make them particularly useful for determining the organization of decision-making circuits (Kristan and Gillette, 2007). In the leech, feeding abolishes all mechanically elicited behaviors including swimming (Misell et al., 1998). Finding the neuronal basis for the decision to feed rather than to produce incompatible behaviors is tractable within the leech nervous system and may reveal how distributed and modular decision-making circuits are organized in general. We have shown that, as leeches begin to feed, descending pathways inhibit the release of transmitter from the presynaptic terminals of the pressure mechanosensory neurons (P-cells) within the segmental ganglia of the nerve cord (Gaudry and Kristan, 2009). This inhibition of sensory input explains why swimming fails to occur during ingestion, but this mechanism does not explain why swimming remains inhibited long after ingestion is completed (Misell et al., 1998).

In this study, we show that the later stages of feeding use a second mechanism to accomplish long-lasting suppression of leech swimming. Leeches increase their body mass by $>10$-fold during a single blood meal (Lent and Dickinson, 1984; Lent et al., 1988). Mimicking the feeding-induced distention by injecting saline (or even air) into a leech terminates feeding (Lent and Dickinson, 1987) and makes swimming less likely to occur (Groome et al., 1993), presumably 
because of the activation of stretch receptors. To help to coordinate swimming movements, stretch receptors in the dorsal longitudinal muscles are activated during the ventral contraction phase of each swim cycle and ventral stretch receptors (VSRs) are activated during the dorsal contraction (Yu and Friesen, 2004). We propose that similar or the same body wall stretch receptors also turn off swimming when they are strongly activated simultaneously during feeding. These findings show that the inhibition caused by distention differs qualitatively from the inhibition caused by food ingestion, both in the sensory neurons involved and in the circuitry targeted.

\section{Materials and Methods}

\section{Electrophysiological recordings}

We obtained extracellular recordings from leech nerves using standard techniques (Gaudry and Kristan, 2009). In short, the dorsal posterior nerve (DP) of a segmental ganglion was cleaned of any surrounding tissue and sucked into the tip of a recording electrode to form a tight seal. The signals were amplified with an A-M Systems differential amplifier (model 1700). We acquired and analyzed data using custom Matlab software (Data Acquisition Toolbox; The MathWorks). For all experiments, we used standard leech saline solution consisting of the following (in $\mathrm{mm}$ ): $115 \mathrm{NaCl}, 4 \mathrm{KCl}, 1.8 \mathrm{CaCl}_{2}, 1.5 \mathrm{MgCl}_{2}, 10$ glucose, adjusted to a $\mathrm{pH}$ of 7.4 with 10 mM HEPES buffer.

\section{Stimulation protocols}

To stimulate mechanosensory terminals in the skin of leeches, we delivered trains of electric shocks with a hand-held electrode touched to the skin. The terminals of the electrode were 0.008 -inch-diameter silver wire coated with Teflon except at the tip, $\sim 1 \mathrm{~mm}$ apart. The stimulus was a train of $8 \mathrm{~V}, 1 \mathrm{~ms}$ pulses delivered at $10 \mathrm{~Hz}$ using a Grass S88 Stimulator and stimulus isolation unit. Each stimulus pulse activates the terminals of one or two touch-sensitive (T-cells) and pressure-sensitive (P-cells) that have terminals in the region stimulated (Kristan et al., 1982). To be sure that the animals were responding to the electrical stimuli and not to the mechanical touch, we lightly touched the electrodes to the skin, and then paused to allow any response to subside (typically, there was no response) before we delivered the electrical pulses, which were activated by a foot switch. We stimulated DP nerves with the same protocol except that the pulses were $2-3 \mathrm{~V}$, delivered through extracellular suction electrodes on the nerves.

\section{Animal preparations}

Severed nerve cord preparations. We weighed and then anesthetized each leech by submerging it in crushed ice made from leech saline. We severed the nerve cord through a small incision between segments 4 and 5, and then sutured the incision shut using silk thread. We trimmed the edges of each animal's caudal sucker to prevent it from attaching to the surfaces of the recording chambers, a behavior that inhibited swimming. We fed the leeches bovine serum from a Falcon tube sealed with sheep intestine, as previously described (Gaudry and Kristan, 2009). We reweighed each leech after it fed to calculate its percentage body mass increase. To determine the effect of removing ingested serum, we lanced some leeches through their body wall and gut wall in their midregion using a surgical blade (no. 11), monitored their swimming behavior in response to stimulation, and reweighed them.

Semi-intact passive distention preparation. We anesthetized leeches using ice-cold leech saline and, through a dorsal incision from the head down to ganglion 4 , we severed the nerve cord between ganglia 1 and 2 and dissected the DP nerves of ganglia 3 and 4 for extracellular recordings. We then cut the body away from ganglion 4 to ganglion 6 , leaving the gut intact and dissected the exposed part of the gut free from the body wall. We dissected the exposed part of the nerve cord away from the body wall. At this point, we weighed the preparation to determine its initial body mass. We then pinned the dissected leech in the chamber using the denervated body wall of segments 4 and 5 . We cannulated the gut with PE-100 tubing whose end had been flared by heating it with a soldering iron. A long thin section of soft silicone tubing [inner diameter (I.D.), 0.012 inch; outer diameter (O.D.), $0.025 \mathrm{inch}]$ was perforated with a scalpel blade and then glued into the flared tip of the PE tubing. The piece of silicone tubing was then cut so that it stopped at the constriction between the gut and the intestine (in segment 15). We slid the perforated silicone tubing down the gut of the animal and tied the opening of the gut to the flared portion of the PE-100 tubing using no. 6 surgical silk. The perforated silicone tubing allowed us to rapidly (within a few seconds) inject a meal-sized amount of saline into the gut and withdraw it through the cannula. As in the active feeding preparations, we trimmed the caudal sucker to prevent the animals from attaching to chamber surfaces. We attached a syringe to the cannula to control and measure the amount of distention. Because the density of the saline used was $1.00 \mathrm{~g} / \mathrm{ml}$, the increase and decrease in the leech's mass was equal and opposite to the volume change in the syringe.

Eviscerated passive distention preparation. To determine whether the gut contained stretch receptors that influenced behavior, we removed all the gut tissue and injected fluid into the body cavity as the animal swam. To gain access to the gut, we turned a leech inside-out, by first anesthetizing it in ice-cold saline, pinning it out ventral-side-up, and decapitating it between segments 3 and 4 . We then moved it into $5^{\circ} \mathrm{C}$ leech saline containing $8 \%$ ethanol for $10 \mathrm{~min}$ to completely relax its muscles. To turn the leech inside-out, we used a $200 \mu \mathrm{l}$ Pipetteman tip to force its posterior sucker into its body cavity to approximately halfway to the opening at the anterior end. We then removed the pipette tip and used a fire-polished glass capillary tube (O.D., $1.0 \mathrm{~mm}$ ) to complete the inversion. Once inverted, we placed the leech into a dissection tray containing ice-cold saline without ethanol. [One preparation was placed in saline containing $50 \mu \mathrm{M}$ serotonin, which improved the performance and the life span of the preparation, presumably because serotonin is lost from the muscles when they are exposed to saline (Mason and Kristan, 1982).] We secured the leech by pinning several latex strips across it and left the glass capillary tube inside the animal to help secure it. We then removed the entire gut of the animal, taking special care to avoid damaging the nerves as they emerged from the CNS and disappeared into the inside of the body wall. We removed the entire intestine except for the very most posterior portion; we denervated and sutured this piece closed to prevent saline from leaking out the anus. We then turned the leech back to its original (outside-out) state using the glass capillary tube.

We cannulated the eviscerated leech using the same sort of PE-100 tubing/perforated silicone tube described in the previous section, except that the body (rather than the gut) was tied tightly to the cannula. We attached the end of the PE tubing to a syringe filled with saline to inject and remove saline. As before, we sometimes added serotonin $(50 \mu \mathrm{M})$ to the injection saline, which prolonged the life of the preparation but did not change the effects of distention on swimming. As before, we trimmed the posterior sucker of the leech so that it could not attach to the chamber. We allowed the leech to recover for an hour or until they became active on their own, whichever was longer. We then transferred the leech to a $400 \mathrm{ml}$ beaker and suspended it in saline by the cannulation tubing. We stimulated the animal using electrical pulses as previously (see above, Stimulation protocols) and quantified its swimming performance.

\section{Isolated nerve cord preparation}

We used isolated nerve cord preparations to determine whether stretching the nerve cord itself would modify the swimming motor program. To determine how much the nerve cord is stretched inside the animal, we videotaped five leeches to determine their body lengths during resting, swimming, crawling, and after feeding. We then selected a separate, sizematched group of leeches and recorded their maximally elongated and contracted states during crawling, which are the longest and shortest lengths that a normal leech achieves behaviorally. We removed the nerve cords from these leeches, as previously described (Kristan and Calabrese, 1976), consisting of ganglia 2 through the tail brain and placed it into a recording chamber containing $50 \mu \mathrm{M}$ serotonin to promote swimming (Willard, 1981; Hashemzadeh-Gargari and Friesen, 1989). We securely pinned down ganglion 10 and used extracellular electrodes to stimulate and record from its DP nerves. We pinned latex strips across the nerve cord between ganglion 10 and adjacent ganglia to secure it in place. The connective anterior to ganglion 2 and the tail brain were loosely pinned and repinned throughout the experiment to vary the length of the nerve 
A
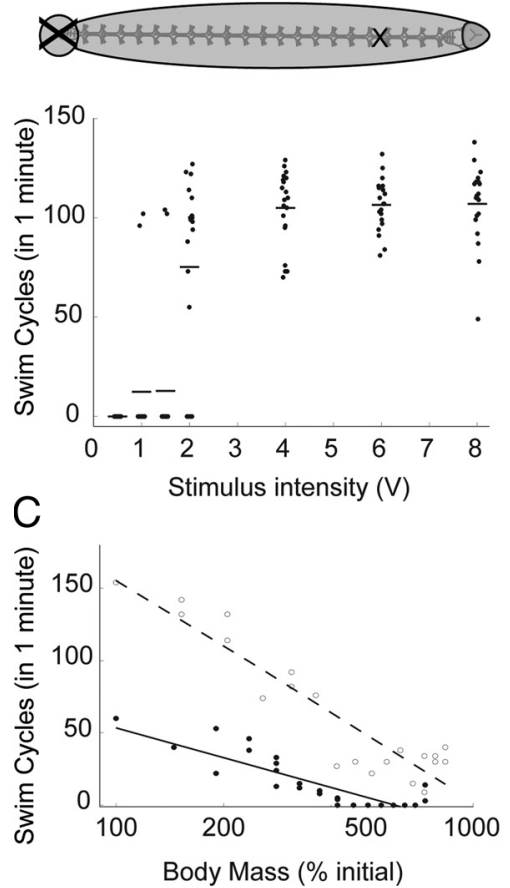

$\mathrm{B}$
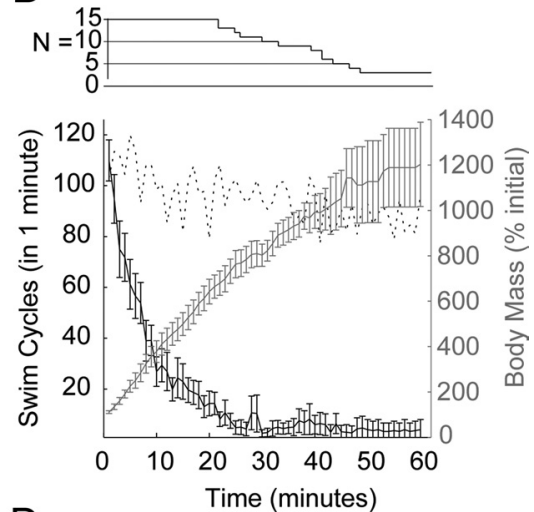

D

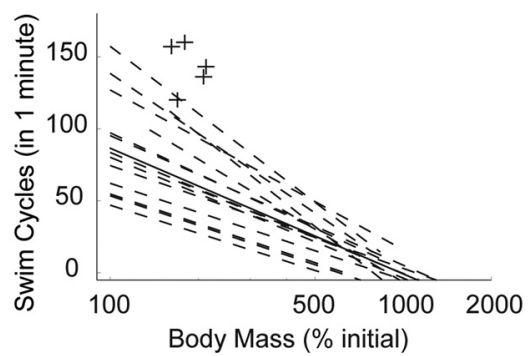

Figure 1. Active feeding increases the body mass in leeches and decreases the number of swim cycles. $\boldsymbol{A}$, Top, Schematic diagram of a leech used in the active feeding experiments. Through a small incision in the ventral body wall exposing the connectives between ganglia 4 and 5, we cut the nerve cord to remove the descending neural inhibition of swimming during feeding. We trimmed the tail sucker to prevent its attachment to the substrate, which tends to inhibit swimming. Bottom, Five unfed, non-distended leeches with severed nerve cords were stimulated four times at each stimulus intensity and the swim cycles elicited were counted for $1 \mathrm{~min}$. A small horizontal jitter was applied to show otherwise overlapping points. The black bars show the mean at each voltage. $\boldsymbol{B}$, The relationship between the length of a feeding bout, number of swim cycles elicited by stimulation, and the increase in body mass. Leeches were stimulated only while feeding, and the duration of feeding bouts varied between animals. Thus, the number of leeches studied decreases as time increases (top plot). The black line shows the decrease in swim cycles observed within 1 min after stimulation as the feeding progresses. The solid gray line indicates a progressive increase in body mass with duration of feeding, measured as percentage of initial body mass. The dashed black line shows the average number of swim cycles for animals that had their nerve cords severed but were not fed $(N=4)$. C, Data and regression lines for two sample leeches used to generate the data in $\boldsymbol{B}$. The open circles and dashed line represent data from one leech, and the filled circles and solid line represent data from the second one. $\boldsymbol{D}$, Plot of the decrease in number of swim cycles elicited by stimulation as a function of the log of the increase in body mass; regressions of individual leeches are shown as gray dashed lines. The solid black line shows the linear regression for all leeches $\left(N=15\right.$ leeches, 589 trials; $\left.R^{2}=0.60 ; p=1.7 \times 10^{-120}\right)$. The plus symbols $(+)$ indicate data for five leeches that were lanced after feeding.

cord. The maximal length used was determined by the maximal length that the relevant part of the animal achieved during crawling.

\section{Statistics}

Regression analyses were performed on custom Matlab software and using Microsoft Excel. We tested for differences in slope and elevation between preparations (intact preparations compared with semi-intacts and preparations with their gut crop removed) by determining the slope and $y$-intercept at mass equals $100 \%$ for each animal. We then performed a Student $t$ test comparing the slopes and $y$-intercepts (at 100\%) between preparations. A logarithmic fit of the data was used when its $R^{2}$ value was higher than the $R^{2}$ value obtained using a linear fit.

\section{Results}

\section{Distention inhibits the maintenance of swimming}

Previously, we showed that ingestion prevents the initiation of swimming in leeches by decreasing the synaptic release from mechanosensory P-cells onto its postsynaptic partners, including the swim trigger neuron Tr1 (Gaudry and Kristan 2009). To test for additional mechanisms that suppress swimming as the leech becomes satiated, we removed the descending inhibition onto P-cells during feeding by severing the connectives of the nerve cords be- tween midbody ganglia 4 and 5 in otherwise intact leeches (Fig. 1A, top). To calibrate our stimulation protocol, we started by stimulating nonfeeding leeches. We stimulated five nonfeeding, severed nerve cord preparations over a range of intensities and measured the number of swim cycles elicited within $1 \mathrm{~min}$. [Leeches do not require the anterior brain to generate normal swimming behavior; in fact, removal of this brain leads to more reliable swimming behavior (Brodfuehrer and Friesen, 1986).] We found that these preparations did not increase their responses at stimulus voltages $>4 \mathrm{~V}$ (Fig. $1 A$, bottom), suggesting that small variations in the positioning of the stimulating electrode would not likely cause large differences in the animal's behavior. We used a stimulus intensity of $8 \mathrm{~V}$ for all subsequent body wall stimulations because it produced a consistent behavioral output and was well above the $2 \mathrm{~V}$ threshold at which some preparations stopped responding.

In severed nerve cord preparations, the anterior end fed normally, whereas the posterior end produced swimming movements in response to skin stimulation. We fed the leeches from an inverted Falcon tube fitted with sausage casing and monitored the quantity of serum consumed per minute. To initiate swimming, we stimulated the rear end of the leeches electrically (Kristan et al., 1982) and counted the number of swim cycles produced before the next stimulus, which was delivered 1 min later. As feeding progressed, the number of elicited swim cycles per minute decreased (Fig. $1 B$, solid black trace) and the average mass of the animals increased (gray trace). To rule out the possibility that habituation or exhaustion caused this decreased swimming, we exposed four animals to the same surgical and stimulation procedures but did not allow them to feed. The number of swim cycles elicited by each electrical stimulus decreased slightly over the hour (Fig. $1 B$, dashed line) but remained significantly higher than the number of cycles produced by feeding animals. Hence, habituation or rundown accounted for only a minor portion of the decreased swimming. To determine the correlation between increases in body mass and the number of swim cycles, we replotted the swim cycles per minute as a function of body mass. Figure $1 C$ shows the data and regression line for two such preparations (one leech is shown with open circles and a dashed regression line and the other is plotted with filled circles and a solid regression line). The leeches with severed nerve cords showed a robust decrease in elicited swim cycles with increasing body mass (Fig. $1 D$; the solid black line shows the mean regression line for the data from 15 leeches shown as dashed gray lines).

To determine whether the distention of the animal caused the decrease in the number of swim cycles produced, we performed two additional experiments. First, we fed five leeches to satiation, and then lanced them (by cutting through the body wall and into the gut using a scalpel) to drain them of much of their ingested 

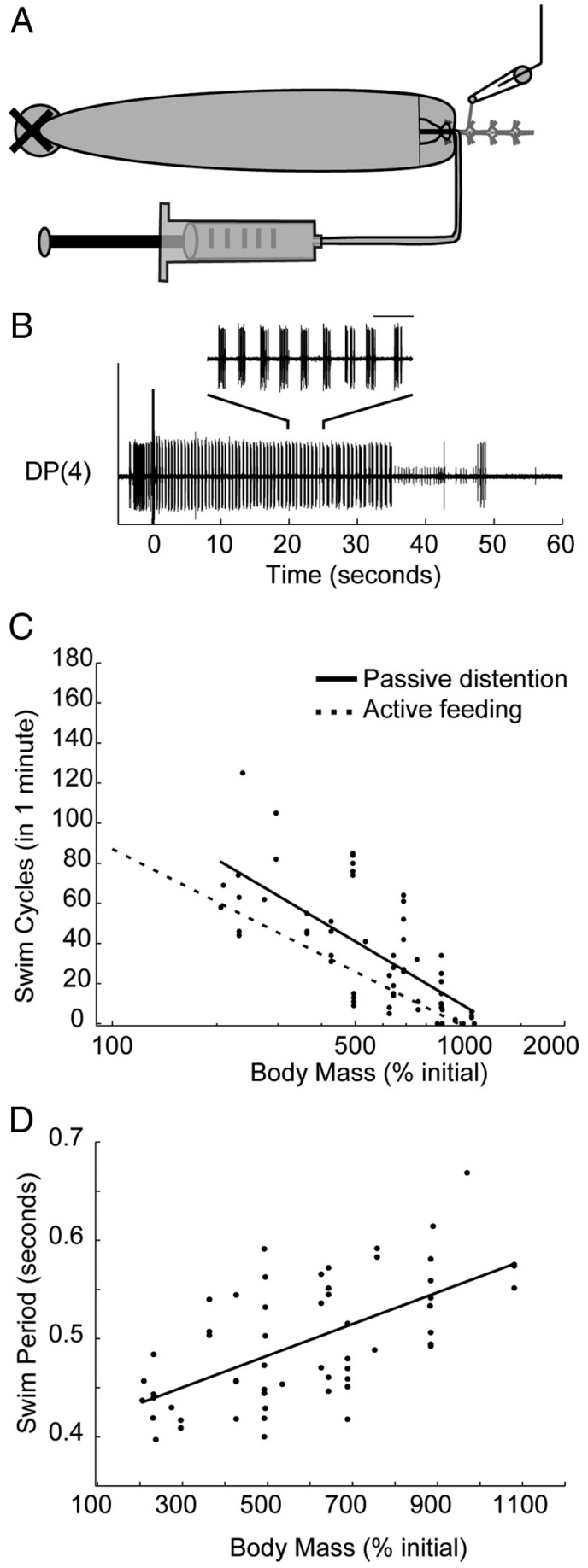

Figure 2. Passive distention reduces the number of swim cycles in response to stimulation in a semi-intact preparation. $\boldsymbol{A}$, Schematic diagram of the semi-intact preparation used for these experiments. We severed the nerve cord between ganglia 2 and 3 and made extracellular recordings from the DP nerves of ganglion 3 or 4 . We then inserted a long perforated tube into the gut of the intact part of the animal and attached it to the open end of the gut with silk sutures (see Materials and Methods, Semi-intact passive distention preparation). Saline solution was injected via a syringe to monitor the amount of distention in the preparation. $\boldsymbol{B}$, Sample trace of a DP nerve recording showing the motor neuron bursts that define swimming. The horizontal bar above inset trace corresponds to $1 \mathrm{~s}$. Between each pair of bursts, the intact portion of the leech swam one complete cycle. At zero time, we stimulated the body wall electrically, as indicated by the large stimulus artifact. Motor activity that precedes the stimulus is from contact made from the stimulating electrode onto the body wall before stimulation. We counted all swim bursts within 1 min after stimulation. The inset shows an expanded view of the swim bursts between 20 and 25 s within the swim episode. $C$, The effect of induced distention on the number of swim cycles observed within $1 \mathrm{~min}$ of stimulation. The $x$-axis is a logarithmic scale because this relationship appeared to be exponential. The black line is the linear regression for these serum meal. The lancing decreased their body mass and returned the number of stimulus-induced swim cycles to slightly above the levels previously found for the prelancing body weight (Fig. $1 D$, plus symbols). This result eliminates the possibility that longterm inhibition of the swim circuit is mediated by exposure of the leech gut to a serum meal. In a second set of experiments, we used a semi-intact preparation in which we could inflate the gut artificially, without the leech needing to feed (Fig. 2A). We removed all the body anterior to ganglion 6 , leaving only the nervous system intact. We left intact the DP nerves on either ganglion 3 or 4 to monitor swim motor activity. We inserted a perforated cannula into the gut remaining in the intact part of the body (see Materials and Methods, Semi-intact passive distention preparation). Using a syringe filled with saline attached to the cannula, we could add measured amounts of saline to the gut and remove it repeatedly. The posterior sucker was deinnervated to keep it from attaching to the chamber. We then stimulated electrically near the tail and monitored the number of swim cycles produced (Fig. 2B). Each DP burst was accompanied by a swim cycle in the intact part of the leech. When we increased the distention levels by injecting saline into their guts, these leeches decreased the number of swim cycles that they produced in response to stimulation (Fig. 2C). The linear regression for such preparations (solid line) is not significantly different from the regression line in actively feeding leeches (dashed line; $p=0.25$ for the slope, and $p=0.17$ for the $y$-intercept at body mass $100 \%$ initial).

Distention also affected the period of swim cycles, as calculated from the bursts of cell 3 action potentials in the DP nerve. The swim cycle period typically increases (i.e., swimming slows down) as the swim episode progresses in fully intact leeches as well as in semiintact preparations and isolated nerve cords (Kristan and Calabrese, 1976). Thus, we compared similar parts of swim episodes across different conditions. Because the number of swim cycles per episode was small in fully distended leeches, we used the burst period during just the first $5 \mathrm{~s}$ of each trial. We found that increases in distention significantly increased the swim period (Fig. 2D).

To determine how quickly distention inhibits the swim central pattern generator (CPG), we rapidly increased the distention of the intact part of the semi-intact preparation. To do this, we first initiated swimming in a semi-intact leech while its gut was empty and then rapidly filled the gut with saline (Fig. 3). From the previous experiments, we knew that swim episodes in nondistended leeches lasted 10-60 s (e.g., the unfed controls in Fig. $1 \mathrm{~B}$ swam for most of the $60 \mathrm{~s}$ interstimulus period). When distended to $\sim 800 \%$ of initial body volume, the semi-intact animal stopped swimming within $\sim 3 \mathrm{~s}$ (Fig. 3A). (We could not get a precise measure of onset time because it typically took $2-3 \mathrm{~s}$ to pass the fluid into the animal slowly enough to avoid rupturing its gut.) Often, swimming spontaneously resumed several seconds after the release of distention. The effect of distention was robust, and it did not habituate even after many repetitions of distention and release of distention (Fig. 3B). In this set of trials, we gave 10 stimuli, with distension trials alternating with non-distension trails. Averaging across four animals showed that swim termination began within $1 \mathrm{~s}$ of distention onset and that all leeches had

$\leftarrow$

data points. The dashed gray line shows best fit derived from active feeding preparations (Fig. 5 ) for comparison $\left(N=3\right.$ leeches, 61 trials; $\left.R^{2}=0.49 ; p=2.9 \times 10^{-10}\right)$. $D$, The swim period increases with increased distention. The swim period was measured as the time interval between the median spikes in sequential bursts. Periods were measured within only the first $5 \mathrm{~s}$ of each trial and averaged $\left(N=3\right.$ leeches, 57 trials; $\left.R^{2}=0.38 ; p=3.2 \times 10^{-7}\right)$. 


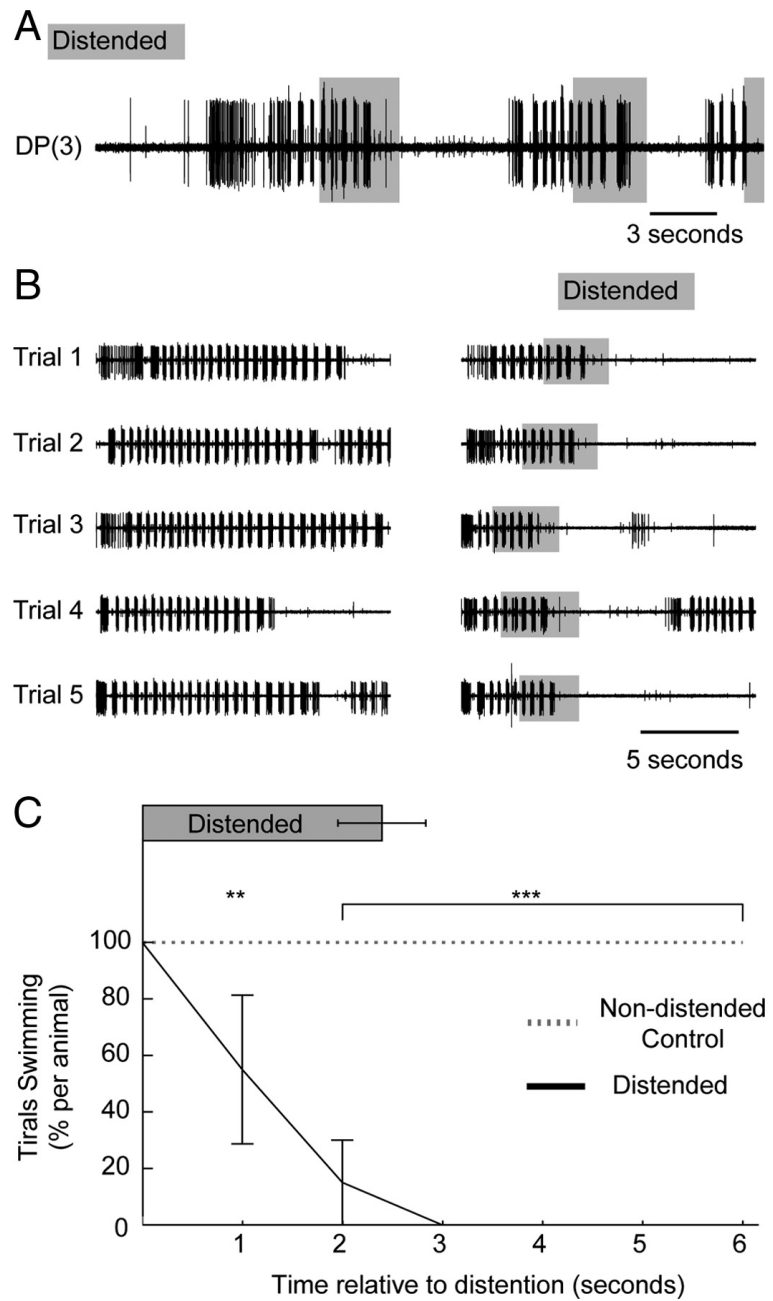

Figure 3. Distention of the leech gut inhibits ongoing swim episodes. $\boldsymbol{A}$, Nerve recording from DP(3) of a spontaneously swimming semi-intact leech. Once the animal was swimming, we injected saline into the gut cavity rapidly until swimming ceased (gray boxes). The volume of saline injected distended the leeches to $\sim 800 \%$ of their initial body mass. Once the saline was removed, swimming often spontaneously resumed some seconds later. $\boldsymbol{B}$, Sample trials from one animal showing the effects of distention on the length of swim episodes. We delivered electrical stimuli at the onset of the recording. Trials alternated between non-distended controls and trials in which saline was injected in the gut of leeches during swimming. The traces on the left show swim episodes in which saline was not injected. The traces on the right show that swim episodes terminated after saline was injected. The gray boxes over each trace show the time of distention. In some trials (e.g., trial 4), swimming resumed after the distention was relieved. C, Summary of the effects of distention on swim episodes. For each time point, the percentage of trials in which swimming had not yet ceased was first calculated and averaged within each animal. These data were then averaged across animals and plotted as a function of time relative to the presentation of the distention. Zero time represents the onset of distention for each distention trial (black line) and the same time point relative to the first swim motor burst in the non-distended trials (dashed gray line). Because the duration of the distention varied somewhat between trials and animals, we plotted the average time of distention \pm SEM. Distention produced a rapid suppression of swim bursts that lasted as long as the distention was maintained and for many seconds after it was relieved (ANOVA with Bonferroni's post hoc test; $F=31.25 ;{ }^{* *} p<0.01,{ }^{* * *} p<0.001 ; N=4$ ). The dotted line is data from nondistended trials: all leeches continued to swim for the $6 \mathrm{~s}$ period observed.

stopped swimming by $3 \mathrm{~s}$ (Fig. $3 C$ ). Swimming continued for $>6$ $s$ in all non-distension trials (dotted line).

\section{Location of distention receptors}

To determine the possible location of the distention receptors, we considered three possible sites: within the CNS, in the lining of
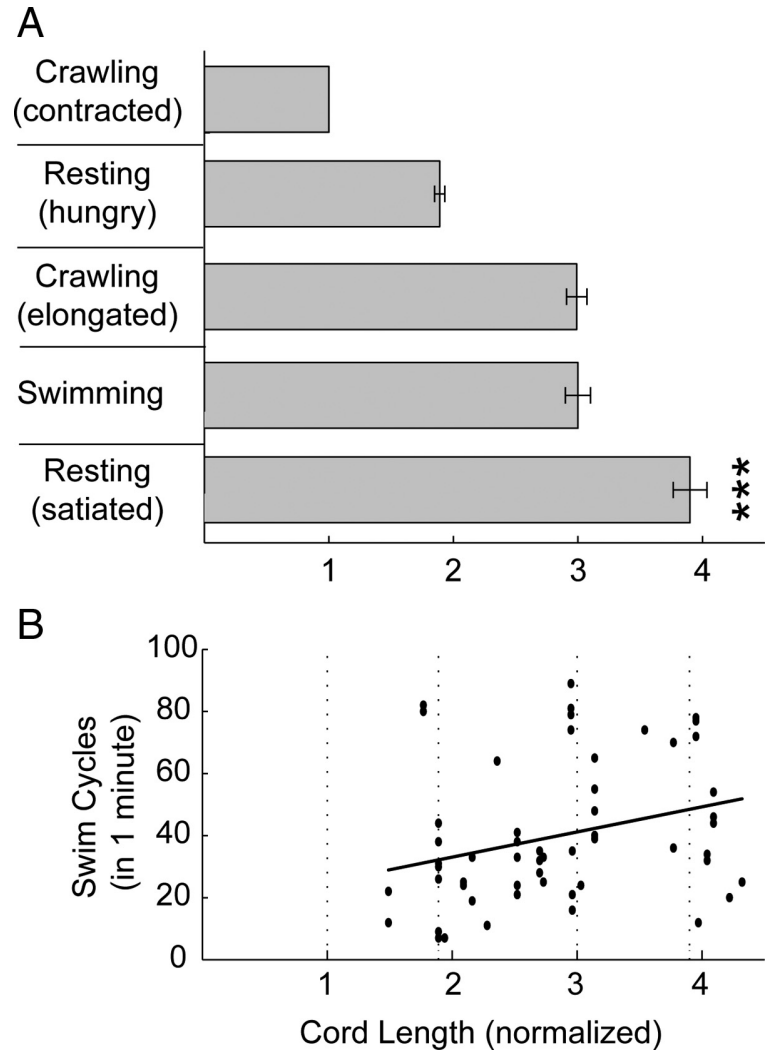

Figure 4. Stretching the leech nerve cord increases the duration of swim bouts. $A$, The bars show the relative lengths of intact leeches as they performed various behaviors, normalized to each leech's minimum length achieved during the contraction phase of crawling. We measured the maximum and minimum lengths achieved in videotapes of hungry leeches as they swam, crawled (maximum and minimum lengths), and at rest (both hungry and satiated). The lengths of the nerve cords were estimated as the length between the middle of their front sucker and the middle of their posterior sucker $(N=5)$. The shortest length achieved in unfed animals was during the contraction phase of crawling, and the longest was during the elongation phase. Fully sated leeches were longer - even at rest - than was ever achieved by hungry leeches (ANOVA with Bonferroni's post hoc test comparing satiated leeches at rest to all other conditions, ${ }^{* * *} p<0.001$, compared to all other bars; $N=4$ ). Error bars indicate SEM. $\boldsymbol{B}$, Relationship between the length of the leech nerve cord and the number of swim cycles elicited by DP nerve stimulation. Data points indicate 58 trials in four leeches $\left(R^{2}=0.084 ; p=0.027\right)$. Cord lengths were normalized to each intact animal's contracted length. The vertical dashed lines show the mean cord lengths for animals during the five behavioral states shown in $\boldsymbol{A}$.

the gut itself, or in the body wall surrounding the gut. We first tested whether the stretch receptors are located within the CNS.

\section{Stretching the nerve cord}

The leech nervous system is highly elastic and stretches as the animal elongates during behaviors such as swimming and crawling (Wilson et al., 1996). To determine how much the nerve cord stretches during various behaviors, we videotaped leeches as they crawled, rested, swam, and fed. We then determined the extremes of body length in each behavior by measuring from the middle of the anterior sucker to the middle of the posterior sucker, which are the locations of the head and tail brains (Fig. 4A). We normalized each animal's body lengths to its most contracted length during crawling. Before feeding, the average maximal length was 3.0 times the fully contracted length, a length achieved both during swimming and during the extension phase of crawling. After feeding to satiation (which, as seen in Fig. $1 B$, produces an average of a 12-fold increase in body mass), even at rest, leeches were 3.9 times longer than their original contracted state, which is significantly longer than they achieved in either swimming or crawling before feeding (Fig. $4 B$ ). Hence, to be 
sure that we mimicked the most extreme feeding-induced length change, we stretched the isolated nerve cord up to 4.5 times its length in the contracted intact leech.

To perform these experiments, we removed the entire CNS, minus the anterior brain (to facilitate swimming in the isolated nervous system), and then stimulated one DP nerve in ganglion 10 and recorded from the contralateral nerve to monitor swimming (Kristan and Calabrese, 1976). We pinned down the isolated nerve cord at ganglion 10 and stretched the two ends of the nerve cord at different lengths. We then initiated swimming episodes and measured the number of swim cycles elicited within 1 min of stimulation. Varying the cord length over the 1.5-4.5 $\times$ range produced a small but significant change in the number of swim cycles elicited (Fig. $4 B)\left(R^{2}=0.084 ; p=0.027 ; N=4\right.$ leeches, 58 trials). The effect of stretching the nerve cord was, however, opposite to that seen during feeding: rather than decreasing swim cycle number, stretching the nerve cord slightly increased the number of swim cycles elicited. The dashed vertical lines in Figure $4 B$ correspond to the mean cord lengths of intact animals engaged in the contraction phase of crawling, at rest, engaged in the elongation phase of crawling, swimming, or at rest after feeding (data taken from Fig. 4A). These data show that stretching the leech CNS does not likely contribute to swim inhibition by distention.

Distending the body wall in leeches with their gut removed To determine whether stretch receptors in the wall of the gut were responsible for sensing distention, we removed the gut from leeches and tested how swimming was affected by distending the remaining body wall. To get access to the gut, we removed the anterior end of the leech through segment 4 and then turned the leech inside-out by pushing the tail sucker through the leech's internal cavity and out through the severed anterior end (Fig. 5A, left). We then dissected away the entire exposed gut of the animal (Fig. 5A, right) and returned the leech to its normal outside-out condition by pushing the tail sucker back through the animal. To complete the preparation, we cannulated the cut anterior portion of the leech. After a recovery period of $\sim 1 \mathrm{~h}$, we stimulated the eviscerated leeches to swim and counted their swim cycles. Injecting fluid through the cannula to distend these animals decreased the number of swim cycles in proportion to the volume of fluid injected (Fig. $5 B$; the solid line is best fit line to the data points). The linear regression for data from these eviscerated animals differed from the data obtained from more intact animals (Fig. $5 B$, dashed line) in their elevation $(p=0.02)$, but not in their slope $(p=0.07)$. This difference indicates that evisceration reduced the overall amount of swimming in these preparations. In fact, given the trauma they experienced in being eviscerated and the fact that the injected saline could go into recesses of the body cavity not available to fluid taken into an intact gut, we find it surprising that the eviscerated animals swam at all, and are impressed that the regression line for the eviscerated animals is so similar to that for the more intact animals.

It is important to note that we cannot rule out the possibility of additional stretch receptors located in the gut of the leech that help to turn off swimming. A test for such gut stretch receptors would necessitate removing the body wall without disturbing gut innervation. Unfortunately, because the nerves from the ganglion pass through the body wall before getting close to the gut, such an experiment is impossible. Nevertheless, the results from stretching the nerve cord and distending eviscerated leeches show that there are peripheral stretch receptors located in the body wall
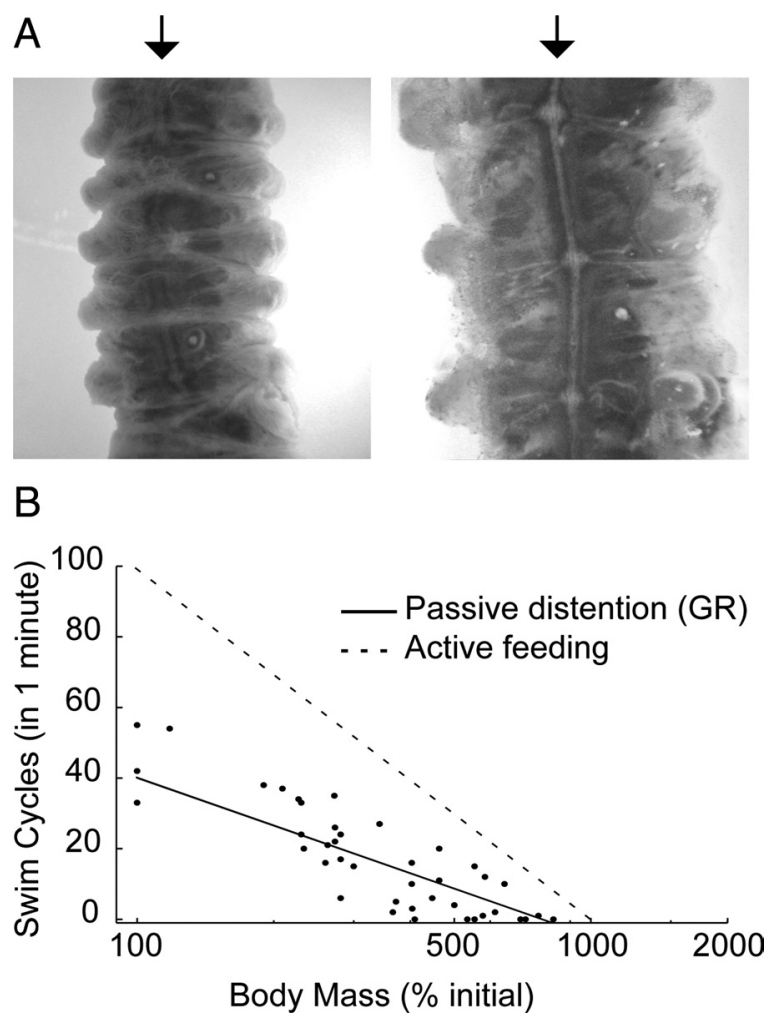

Figure 5. Removing the gut of the leech does not eliminate the distention-induced suppression of swimming. $A$, Left, Photograph of a leech turned inside-out with the gut intact. The black arrow points to the CNS, which is covered by the gut. $A$, Right, Photograph of the same insideout leech after all the gut tissue has been removed. The CNS (indicated by black arrow) and peripheral nerves are more readily visible. $\boldsymbol{B}$, The effect of induced distention on the number of swim cycles elicited by DP nerve stimulation in eviscerated leeches. The swim cycles in leeches with guts removed [passive distention (GR)] decreases as percentage initial body mass increases $\left(43\right.$ trials from 4 leeches; $R^{2}=0.64 ; p=9.68 \times 10^{-11}$ ). The solid black line shows the linear regression for the data points shown and the dashed gray line is the regression line for data obtained from intact leeches (Fig. 1C). These two lines differ in $y$-intercept at body mass (percentage initial) but not in slope ( $p=0.02$ and $p=0.07$, respectively).

that respond to distention and inhibit swimming, and no central stretch receptors located in the nerve cord.

\section{Discussion}

Extending previous observations that body distention inhibits feeding (Lent and Dickinson, 1987) and decreases the probability of swimming (Groome et al., 1993), we have shown that the prolonged inhibition of swimming after feeding is proportional to the distention of the leech's body (Figs. 1,2), the inhibition is expressed within seconds (Figs. 3, 4), and the inhibition probably results from activating peripheral stretch receptors (Figs. 5, 6). Together with previously published results showing presynaptic inhibition as a mechanism by which feeding inhibits swimming (Gaudry and Kristan 2009), these new findings show that leeches use different mechanisms to turn off swimming during feeding and during postfeeding satiation. In other words, ingestion and distention target different components of the leech swim circuit to disable it both acutely and persistently.

Prolonged inhibition: decision making by maintained stretch Likely candidates for the distention-induced suppression of swimming are the previously described body wall stretch receptors (Blackshaw and Thompson, 1988; Huang et al., 1998) known to be important in shaping leech swimming (Cang and Friesen, 

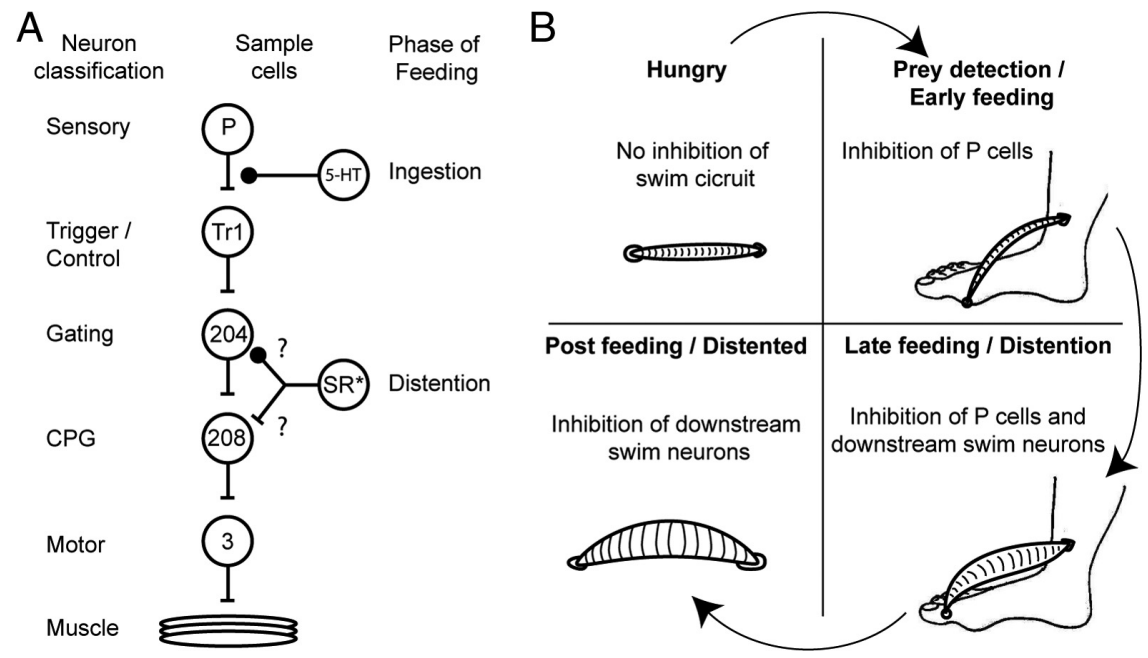

Figure 6. Diagram summarizing the two ways that leech feeding inhibits the swimming circuit. $\boldsymbol{A}$, The circles represent cell populations; the letters and numbers inside the circles indicate one identified neuron from that population type. The lines ending in bars represent excitatory connections, and those ending in solid black circles represent inhibitory connections. The diagram shows the excitatory, feedforward nature of the circuit but does not show the inhibitory interactions among the CPG neurons and between particular motor neurons. The inhibition from ingestion arises from an unknown source, probably chemical or thermal sensory pathways; it inhibits the P-cell terminals. The actions of distention likely originate from stretch receptors in the body wall and target either the gating neurons or CPG neurons. The inhibition of cell 204 is speculative but consistent with an increase in swim period and a cessation of swimming behavior. Because leech stretch receptors hyperpolarize during stretch, the excitation of cell 208 may reflect the removal of inhibition rather than direct excitation. The swim circuit connections have been identified previously (Kristan et al., 2005). P, Pressure mechanosensory P-cell; Tr1, trigger neuron 1; 204, gating neuron 204; 208, CPG neuron 208; 3, dorsal longitudinal muscle excitatory motor neuron $3 ; \mathrm{SR}^{*}$, stretch receptors speculated to encode distention; ?, potential connections. $\boldsymbol{B}$, A schematic model for when each inhibitory mechanism is recruited during feeding. Before feeding, feeding circuitry does not inhibit the leech swim circuit. At the onset of a feeding episode (hungry), ingestion inhibits the P-cells of the leech (prey detection/early feeding). Additional ingestion leads to distention and the recruitment of inhibition of swim neurons downstream of the P-cells (late feeding/distention). After feeding, P-cells are relieved of the presynaptic inhibition and behaviors compatible with the distended state return online (post feeding/distended). Swimming remains inhibited by distention-mediated targeting of downstream swim neurons, and the leech is biased toward crawling.

2000; Yu and Friesen, 2004). These stretch receptors are located in the body wall, closely applied to the longitudinal muscle layer. They are stretched and hyperpolarized whenever the animal elongates. The VSR makes synaptic contact with motor neurons and interneurons responsible for generating the swim rhythm (Cang et al., 2001). Stretching the VSR during the dorsal phase of swimming hyperpolarizes motor neurons and interneurons that are active in the dorsal contraction phase of swimming and depolarizes neurons that are active in the ventral phase. Although less complete, studies conducted on the dorsal stretch receptor (DSR) suggest that they have opposite effects on the leech swim circuit (Fan and Friesen, 2006), working in unison with the VSR to provide a more robust rhythm. After a full meal, even leeches at rest are longer than they were during swimming or crawling when unfed (Fig. 4A). If these stretch receptors are responsible for the prolonged inhibition produced by distention, their inhibitory effects must predominate when the VSR and DSR are simultaneously activated.

The increase in swim period that we observed during distention can be mimicked by injecting hyperpolarizing current into the swim gating neuron, cell 204 (Weeks and Kristan, 1978). The endogenous firing rate of cell 204 is also highly correlated with the period of swimming (Debski and Friesen, 1986). The gating cells provide excitation for the central pattern generator, which in turn drives the motor neurons (Weeks, 1982; Nusbaum et al., 1987). Hence, a reasonable hypothesis for how distention might turn off swimming is by hyperpolarizing cell 204 and thus increasing the period of swimming and decreasing the likelihood of swim initiation (Fig. 6A).

\section{Behavioral switching to crawling after feeding}

Many intrinsic and extrinsic factors influence a leech's decision to swim, including the depth of water surrounding the leech (Esch et al., 2002), the level of various neuromodulators percolating through the CNS (Crisp and Mesce, 2003, 2006), and the satiation level of the leech (Misell et al., 1998). After feeding, leeches crawl, rather than swim, away from tactile stimulation. The switching from swimming to crawling observed after feeding could be the result of changes to cell 208, a neuron critical in the decision of whether to swim or crawl in response to stimulation (Briggman et al., 2005). Cell 208 is an unusual member of the swim circuit: it is the only excitatory member of the swim CPG and functions to connect the swim gating neurons to the swim CPG (Weeks, 1982; Nusbaum et al., 1987; Kristan et al., 2005). Cell 208 is active during the dorsal phase of swimming, but unlike other cells active in the same phase, it depolarizes in response to ventral stretch in contrast to other cells, which hyperpolarize (Cang et al., 2001). Furthermore, cell 208 is the only CPG neuron likely to have a direct synaptic connection to the VSR; all the other CPG neurons have clearly polysynaptic connections to the VSR (Cang et al., 2001). Hyperpolarization of the VSRs, as might occur during distention, is sufficient to depolarize cell 208 and depolarization of cell 208 biases leeches to crawl in response to DP stimulation (Briggman et al., 2005). Thus, it is possible that distention not only inhibits swimming by inhibiting swim-related neurons but also promotes crawling by targeting and depolarizing the decision-making neuron 208 (Fig. 6A).

\section{The inhibition of swimming during feeding is distributed and modularized}

Feeding in leeches appears to inhibit the swim circuit in both a distributed and modularized manner (Fig. 6A). The decision to stop swimming is distributed in that it most likely arises from two distinct sources: one that generates ingestion, and the other that is activated by distension. We have shown previously that ingestion activates a descending pathway that targets the pressure mechanosensory neurons (P-cells) of the leech (Gaudry and Kristan, 2009). This descending inhibition decreases the probability of release measured at presynaptic release sites of the P-cells. This current study (Fig. 1) further illustrates this point by showing that severing the nerve cord is sufficient to relieve posterior segments of this inhibition and allows swimming to occur simultaneously with feeding. Hence, the inhibition of the leech swim circuit by distention must originate from a different source, most likely the stretch receptors located within the body wall musculature of the animal. Unlike ingestion, distention targets components of the swim circuit downstream of the sensory neurons.

Because ingestion and distention have two distinct sources and targets for their inhibition, we can think of them as unique decision-making modules. Why have two different modules to 
inhibit swimming during feeding? The two mechanisms do not appear to be redundant but are better described as complementary. Ingestion prevents the initiation of swimming, and distention inhibits the maintenance of swimming. More importantly, however, the effects of ingestion are to broadly inhibit all mechanically elicited behaviors, whereas distention leaves some behaviors intact while suppressing others. By segregating different components of the decision-making process into separate modules, the leech can recruit either strategy independent of the other (Fig. 6B). For example, during the appetitive and ingestion phase of feeding, it is important to suppress all mechanically elicited behaviors that may compete and interfere with ingestion. Thus, direct inhibition of the mechanoreceptors is an ideal strategy to prevent competing behaviors from being expressed. The inhibition of mechanosensory receptors should also allow the leech to perform spontaneous behaviors that facilitate feeding. For example, even though leeches fail to respond to stimulation while exploring a feeding site (Misell et al., 1998), they must remain able to locomote to find a suitable site to begin feeding. By targeting sensory input, this mechanism prevents external stimuli from interfering with feeding but allows for the generation of spontaneous behaviors. During early ingestion, we sometimes observe sinusoidal swim-like behavior during feeding (our personal observation). Although this behavior appears very much like swimming, it differs in two qualitatively important manners. First, the front sucker always remains attached to the food source. Second, this behavior is never elicited through mechanical stimulation in a variety of sanguivorous leech species, including Hirudo (Misell et al., 1998; Gaudry et al., 2010). This oscillatory behavior appears to distribute blood throughout the gut and illustrates a behavior that is "spontaneously" generated during feeding (i.e., it is not caused by mechanosensory stimulation) and does not interfere with feeding. As ingestion continues, the leech remains insensitive to external touch and begins to display distention-induced suppression of swimming. External stimuli do not elicit swimming in this state. During the experiments performed in Figure 1, we monitored the occurrence of spontaneous swim episodes between stimulation periods (data not shown). Although these swim episodes were quite rare to begin with, interstimulus swimming never occurred with increasing distention.

Once the leech is sated, ingestion is terminated in part through distention (Lent and Dickinson 1987). After a large meal, leeches reliably locomote away from their food source by crawling rather than swimming (Misell et al., 1998). The inhibition of P-cell terminals quickly dissipates after ingestion, so the leech regains its responsiveness to mechanical stimulation and can generate locomotory behavior, either in response to stimulation or spontaneously. The distention acquired during feeding, however, serves to suppress swimming in the postfeeding state and biases the leech toward crawling when attempting to flee its host.

In the mammalian brain, decision-making circuits have been shown to employ both distributed circuits and modular circuitry. For instance, during a flutter discrimination task, many cortical regions show neuronal correlates time-locked to two different stimuli (Romo and Salinas, 2003), showing their distributed nature. In these experiments, monkeys received two successive vibrating tactile stimuli and they needed to report whether the second stimulus was higher in frequency or lower than the first. Some cortical regions play a clear role in this task such as stimulus coding in primary somatosensory cortex (S1) and motor output coding in primary motor cortex (M1). In addition, neuronal correlates of more cognitive aspects of the task, such as storing the frequency of the first stimulus or comparing the two stimuli, was found within either the prefrontal cortex (PFC), the secondary somatosensory cortex (S2), or the medial premotor cortex (MPC). What differs between these areas is a large shift in distribution of response types. Experiments recording from monkeys trained to make visual discriminations have also suggested a distributed circuitry. The decision to make goal-oriented saccades depends on several brain regions, each performing its own part: superior colliculus neurons show correlates of when and where to saccade (Glimcher and Sparks, 1992); LIP neurons are correlated with the probability of receiving a reward for an action (Platt and Glimcher, 1999; Glimcher, 2003); and basal ganglia neuronal activity correlates with updating reward probabilities for a specific saccade (Schultz, 2000; Schultz and Dickinson, 2000). Vibrotactile and visual discrimination studies have also suggested modular decision-making circuits that play the same role in both tasks. For example, the PFC appears to play the same role of storing information about either tactile or visual stimuli in working memory (Miller et al., 1996; Romo et al., 1999). These studies suggest that decision-making circuits are both distributed and modular, in mammals as well as in leeches, permitting animals to combine or express different decision-making modules as the task or environment changes.

\section{References}

Blackshaw SE, Thompson SW (1988) Hyperpolarizing responses to stretch in sensory neurones innervating leech body wall muscle. J Physiol 396:121-137.

Briggman KL, Abarbanel HD, Kristan WB Jr (2005) Optical imaging of neuronal populations during decision-making. Science 307:896-901.

Brodfuehrer PD, Friesen WO (1986) Control of leech swimming activity by the cephalic ganglia. J Neurobiol 17:697-705.

Cang J, Friesen WO (2000) Sensory modification of leech swimming: rhythmic activity of ventral stretch receptors can change intersegmental phase relationships. J Neurosci 20:7822-7829.

Cang J, Yu X, Friesen WO (2001) Sensory modification of leech swimming: interactions between ventral stretch receptors and swim-related neurons. J Comp Physiol A Neuroethol Sens Neural Behav Physiol 187:569-579.

Crisp KM, Mesce KA (2003) To swim or not to swim: regional effects of serotonin, octopamine and amine mixtures in the medicinal leech. J Comp Physiol A Neuroethol Sens Neural Behav Physiol 189:461-470.

Crisp KM, Mesce KA (2006) Beyond the central pattern generator: amine modulation of decision-making neural pathways descending from the brain of the medicinal leech. J Exp Biol 209:1746-1756.

Debski EA, Friesen WO (1986) Role of central interneurons in habituation of swimming activity in the medicinal leech. J Neurophysiol 55:977-994.

Esch T, Mesce KA, Kristan WB (2002) Evidence for sequential decision making in the medicinal leech. J Neurosci 22:11045-11054.

Fan RJ, Friesen WO (2006) Characterization of central axon terminals of putative stretch receptors in leeches. J Comp Neurol 494:290-302.

Gaudry Q, Kristan WB Jr (2009) Behavioral choice by presynaptic inhibition of tactile sensory terminals. Nat Neurosci 12:1450-1457.

Gaudry Q, Ruiz N, Huang T, Kristan WB 3rd, Kristan WB Jr (2010) Behavioral choice by across leech species: chacun a son gout. J Exp Biol 213:1356-1365.

Glimcher PW (2001) Making choices: the neurophysiology of visualsaccadic decision making. Trends Neurosci 24:654-659.

Glimcher PW (2003) The neurobiology of visual-saccadic decision making. Annu Rev Neurosci 26:133-179.

Glimcher PW, Sparks DL (1992) Movement selection in advance of action in the superior colliculus. Nature 355:542-545.

Groome JR, Clark M, Lent CM (1993) The behavioural state of satiation in the leech is regulated by body distension and mimicked by serotonin depletion. J Exp Biol 182:265-270.

Hare TA, O’Doherty J, Camerer CF, Schultz W, Rangel A (2008) Dissociating the role of the orbitofrontal cortex and the striatum in the computation of goal values and prediction errors. J Neurosci 28:5623-5630.

Hashemzadeh-Gargari H, Friesen WO (1989) Modulation of swimming activity in the medicinal leech by serotonin and octopamine. Comp Biochem Physiol C 94:295-302. 
Huang Y, Jellies J, Johansen KM, Johansen J (1998) Development and pathway formation of peripheral neurons during leech embryogenesis. J Comp Neurol 397:394-402.

Izuma K, Saito DN, Sadato N (2008) Processing of social and monetary rewards in the human striatum. Neuron 58:284-294.

Kandel E, Schwartz J, Jessel T (2000) Principles of neural science, Ed 4. New York: McGraw-Hill.

Kristan WB, Gillette R (2007) Decision-making in small neuronal networks. In: Invertebrate neurobiology (North G, Greenspan R, eds), pp 533-554. Cold Spring Harbor, NY: Cold Spring Harbor Laboratory.

Kristan WB Jr, Calabrese RL (1976) Rhythmic swimming activity in neurones of the isolated nerve cord of the leech. J Exp Biol 65:643-668.

Kristan WB Jr, McGirr SJ, Simpson GV (1982) Behavioural and mechanosensory neurone responses to skin stimulation in leeches. J Exp Biol 96:143-160.

Kristan WB Jr, Calabrese RL, Friesen WO (2005) Neuronal control of leech behavior. Prog Neurobiol 76:279-327.

Lent CM, Dickinson MH (1984) Serotonin integrates the feeding behavior of the medicinal leech. J Comp Physiol A Neuroethol Sens Neural Behav Physiol 154:457-471.

Lent CM, Dickinson MH (1987) On the termination of ingestive behaviour by the medicinal leech. J Exp Biol 131:1-15.

Lent CM, Fliegner KH, Freedman E, Dickinson MH (1988) Ingestive behaviour and physiology of the medicinal leech. J Exp Biol 137:513-527.

Liu X, Powell DK, Wang H, Gold BT, Corbly CR, Joseph JE (2007) Functional dissociation in frontal and striatal areas for processing of positive and negative reward information. J Neurosci 27:4587-4597.

Mason A, Kristen WB Jr (1982) Neuronal excitation, excitation, inhibition and modulation of leech longitutinal muscle. J Comp Physiol 146:527536.

Miller EK, Erickson CA, Desimone R (1996) Neural mechanisms of visual working memory in prefrontal cortex of the macaque. J Neurosci 16:5154-5167.

Misell LM, Shaw BK, Kristan WB Jr (1998) Behavioral hierarchy in the me- dicinal leech, Hirudo medicinalis: feeding as a dominant behavior. Behav Brain Res 90:13-21.

Nusbaum MP, Friesen WO, Kristan WB Jr, Pearce RA (1987) Neural mechanisms generating the leech swimming rhythm: swim-initiator neurons excite the network of swim oscillator neurons. J Comp Physiol A Neuroethol Sens Neural Behav Physiol 161:355-366.

Platt ML, Glimcher PW (1999) Neural correlates of decision variables in parietal cortex. Nature 400:233-238.

Romo R, Salinas E (2003) Flutter discrimination: neural codes, perception, memory and decision making. Nat Rev Neurosci 4:203-218.

Romo R, Brody CD, Hernandez A, Lemus L (1999) Neuronal correlates of parametric working memory in the prefrontal cortex. Nature 99:470473.

Schultz W (1998) Predictive reward signal of dopamine neurons. J Neurophysiol 80:1-27.

Schultz W (2000) Multiple reward signals in the brain. Nat Rev Neurosci 1:199-207.

Schultz W, Dickinson A (2000) Neuronal coding of prediction errors. Annu Rev Neurosci 23:473-500.

Weeks JC (1982) Synaptic basis of swim initiation in the leech. II. A pattern generating neuron cell 208, which mediates motor effects of swiminitiating neurons. J Comp Physiol A Neuroethol Sens Neural Behav Physiol 148:265-279.

Weeks JC, Kristan WBJ (1978) Initiation, maintenance and modulation of swimming in the medicinal leech by the activity of a single neurone. J Exp Biol 77:71-88.

Willard AL (1981) Effects of serotonin on the generation of the motor program for swimming by the medicinal leech. J Neurosci 1:936-944.

Wilson RJA, Skierczynski BA, Meyer JK, Skalak R, Kristan WB (1996) Mapping motor neuron activity to overt behavior in the leech. J Comp Physiol A Neuroethol Sens Neural Behav Physiol 178:637.

Yu X, Friesen WO (2004) Entrainment of leech swimming activity by the ventral stretch receptor. J Comp Physiol A Neuroethol Sens Neural Behav Physiol 190:939-949. 